\title{
BAALC Gene Expression in Adult B-precursor Acute Lymphoblastic Leukemia: Impact on Prognosis
}

Mona M. Taalab1, Iman M. Fawzy ${ }^{2 *}$, Enas F. Goda ${ }^{3}$ and Eman M. Abdul Salam 4

${ }^{1}$ Department of Clinical Hematology, Internal Medicine, Faculty of Medicine, Mansoura University, Mansoura, Egypt ${ }^{2}$ Department of Laboratory Medicine, Mansoura Fever Hospital, Ministry of Health, Mansoura, Egypt

${ }^{3}$ Departments of Clinical Pathology, Faculty of Medicine, Mansoura University, Mansoura, Egypt

${ }^{4}$ General Medicine, Faculty of Medicine, Azhar University, Cairo, Egypt

\begin{abstract}
Background: Adult B-precursor acute lymphoblastic leukemia (ALL) remains a major therapeutic challenge. Various molecular markers have extensively been investigated to improve its risk profile characterization, disease progression and resistance to treatment.
\end{abstract}

Aim: To analyze the brain and acute leukemia, cytoplasmic (BAALC) gene expression and to assess its prognostic impact in B-precursor ALL.

Subjects and Methods: BAALC mRNA expression was analyzed using real time PCR in 200 primary adult B-precursor ALL patients. Patients were grouped into 2 groups according to median BAALC expression.

Results: High $B A A L C$ expression was associated with older age $(P=0.037)$, higher white blood cell count $(P=0.019)$, LDH concentration $(p=0.007)$, higher incidence of positive CD34 $(P=0.011)$ and positive $B C R-A B L$ $(P=0.011)$. High $B A A L C$ expression was associated with primary therapy resistance in the overall cohort $(P=$ $0.001)$, in BCR-ABL and BCR-ABL ${ }^{+}$subgroups $(P=0.039,0.003$ respectively). Multivariate analysis showed that $B A A L C$ expression was an independent risk factor for chemotherapy resistance in the overall cohort $(p=0.003$, $\mathrm{OR}=3.133,95 \% \mathrm{Cl}=1.482-6.623)$ and in both BCR-ABL $(p=0.049, \mathrm{OR}=2.359,95 \% \mathrm{Cl}=1.004-5.538)$, and BCR$\mathrm{ABL}^{+}$subgroups $(p=0.014, \mathrm{OR}=2.672,95 \% \mathrm{Cl}=1.824-3.326)$. Higher $B A A L C$ expression was associated with a shorter overall survival (OS) $(p=0.010)$ and disease free survival (DFS) $(p<0.001)$ in the overall cohort, and DFS in BCR-ABL- subgroup $(p<0.001)$. Multivariate analysis showed that higher BAALC expression independently predicted OS and DFS in the overall cohort $(P=0.039,0.002$ respectively) and DFS in BCR-ABL- subgroup $(p=0.001)$.

Conclusion: High BAALC expression is associated with refractory disease in adult B-precursor ALL, and predicts shorter OS and DFS. Determination of BAALC expression may contribute to risk stratification of adult $\mathrm{B}$-precursor ALL, and may improve the currently disappointing cure rate.

Keywords: BAALC; Adult ALL; Leukemia; Refractory; Prognosis

\section{Introduction}

Acute lymphoblastic leukaemia (ALL) is a heterogeneous disease with distinct manifestations and prognostic and therapeutic implications [1]. ALL in adults is a rare disease. The results of therapy remain unsatisfactory, and progress has been relatively slow [2]. B-cell ALL (B-ALL) is a clonal malignant disease originated in a single cell and characterized by the accumulation of blast cells that are phenotypically reminiscent of normal stages of B-cell differentiation [3].

Outcome of adult B-precursor ALL has considerably improved because of identification of clinical and genetic risk factors stratifying patients to different treatment groups [4]. Commonly accepted risk factors in B-precursor ALL include age, performance status, white blood cell (WBC) count, lactate dehydrogenase concentration, the immunophenotype, response to induction therapy, level of minimal residual disease, cytogenetics and genetic aberrations [5,6]. Patients lacking clinical and molecular risk factors are considered standard risk (SR). Outcome for SR patients is still unsatisfactory [5,7] indicating the clinical and biologic heterogeneity of these patients. Therefore, the identification of novel predictive molecular markers in adult B-precursor ALL may improve treatment stratification of this subgroup.

The Brain And Acute Leukemia, Cytoplasmic (BAALC) gene is highly expressed in normal uncommitted progenitor cells and downregulated with the onset of differentiation [8]. An elevated expression of the
$B A A L C$ gene was originally discovered in a gene expression profiling study of acute myeloid leukemia (AML) with trisomy 8 , but was later also found in other AML and in ALL $[9,10]$. Over expression of BAALC predicts an inferior outcome in AML and ALL patients [9-11].

Therefore, our aim in the present study is to analyze BAALC expression and to evaluate its prognostic impact on clinical outcome of adult B-precursor ALL.

\section{Methods}

\section{Patients}

Two hundred newly diagnosed as adult B-precursor ALL were enrolled between 2005 and 2014 admitted to Mansoura Oncology Center, Mansoura, Egypt. Specimens were selected from consecutive

*Corresponding author: Iman M. Fawzy, Department of Laboratory Medicine, Mansoura Fever Hospital, Ministry of Health, Mansoura, Egypt, Tel: 1092600249; E-mail: hematologyaml@yahoo.com

Received April 23, 2014; Accepted May 26, 2014; Published June 06, 2014

Citation: Taalab MM, Fawzy IM, Goda EF, Salam EMA (2014) BAALC Gene Expression in Adult B-precursor Acute Lymphoblastic Leukemia: Impact on Prognosis. J Blood Disorders Transf 5: 220. doi: 10.4172/2155-9864.1000220

Copyright: (c) 2014 Taalab MM, et al. This is an open-access article distributed under the terms of the Creative Commons Attribution License, which permits unrestricted use, distribution, and reproduction in any medium, provided the original author and source are credited. 
Citation: Taalab MM, Fawzy IM, Goda EF, Salam EMA (2014) BAALC Gene Expression in Adult B-precursor Acute Lymphoblastic Leukemia: Impact on Prognosis. J Blood Disorders Transf 5: 220. doi: 10.4172/2155-9864.1000220

patients who had sufficient material available. They were 106 males (53\%) and 94 females ( $47 \%)$ with mean age $42.32 \pm 10.675$ years. Patients were diagnosed according to standard diagnostic methods including clinical, cytomorphological, cytochemical, immunophenotypic methods. In addition, 20 healthy subjects, of matched age $(39 \pm 11.202$ years) and sex (12 males (60\%) and 8 females (40\%)); with normal laboratory findings; were selected as a control group. Informed consent was obtained from all patients. Risk groups were assigned as follows: SR (no risk factor), high risk ( $\geq 1$ risk factor), and very high risk (presence of $\mathrm{t}(9 ; 22) / B C R-A B L)$.

\section{Treatment protocol}

ALL cases were treated according to our risk adapted chemotherapy protocol: The patients were stratified according to their prognostic factors into standard, high, and very high risk groups. The treatment plan included: Prephase for patients with high WBC and/or organomegaly. Induction phase I: Four drugs: Vincristine, Doxorubicin, L-Asparaginase and prednisone with intrathecal MTX. Patients that attained complete remission (CR) were subjected to cranial irradiation with $24 \mathrm{~Gy}$ and intrathecal MTX for four injections. Phase II induction with Cyclophosphamide and Cytarabine. Consolidation phase I: Vincristine, Doxorubicine and prednisone with Triple intrathecal. Phase II consolidation: Cyclophosphamide, Cytarabine and Etoposide with triple intrathecal. Maintenance therapy: two years with 6 mercaptopurine and methotrexate. For patients with high and very high risks who were not planned to stem cell transplantation, one cycle of high dose cytarabine and mitoxantrone (HAM regimen) was added between induction and consolidation. Bone marrow aspirate was done to evaluate response to chemotherapy (status post induction). Cases who died before treatment or who didn't receive treatment due to poor performance status or elderly cases kept on supportive treatment were excluded, as well as patients who received SCT in first CR.

\section{Criteria of response and survival definitions}

Complete remission (CR) was assessed after completion of induction chemotherapy. CR was defined as follows: granulocyte count of at least $1.5 \times 10^{9} / \mathrm{L}$, platelet count of at least $100 \times 10^{9} / \mathrm{L}$, no peripheral blood $(\mathrm{PB})$ blasts, bone marrow $(\mathrm{BM})$ cellularity of at least $20 \%$ with maturation of all cell lines and less than $5 \%$ blasts, and no extramedullary leukemia. Primary therapy failure (refractory disease) was defined as persistence of PB blasts or at least 25\% blasts in BM after induction therapy. Relapse was defined as reappearance of $\mathrm{PB}$ blasts, more than $5 \%$ blasts in $\mathrm{BM}$, or appearance of extramedullary manifestations after CR was achieved. OS was defined as the time from diagnosis to date of death. For patients achieving CR, DFS was the time from the date of first CR to an event (death in first CR or relapse).

\section{Immunophenotypic analysis}

Immunophenotypic analyses were performed by flow cytometry on fresh pretreatment BM and PB samples. A wide panel of monoclonal antibodies (Mo Abs) was used. Lymphoid markers included CD19, $\mathrm{CD} 22$ for B Lineage and CD1, CD2, CD3, CD4, CD5 and CD7, for exclusion of T-lineage and other markers used included CD45, HLADR, CD10, and CD34. All the monoclonal antibodies were obtained from Coulter Hialeah, FL. A cell-surface antigen was considered positive when at least $20 \%$ of cells showed fluorescence intensity greater than the negative control.

\section{Molecular analyses}

Real-time quantitative polymerase chain reaction (RTQ-PCR) for BCR-ABL

To assess molecular responses, total RNA was extracted from $\mathrm{PB}$ or BM blood cells. BCR-ABL and internal control transcript levels were quantified using real-time PCR analysis (TaqMan) on an ABI prism 7000 sequence detection system (Applied Biosystems, Foster City, CA). Specific PCR products were amplified and detected using dual-fluorescent non-extendable probes labeled with 6-carboxyfluorescein (FAM), reporter and 6-carboxytetramethylrhodamine (TAMRA), quencher at $5{ }^{\prime}$-end and $3^{\prime}{ }^{-}$-end, respectively. The relative mRNA expression of BCR-ABL transcript was calculated using the comparative cycle threshold (Ct) method [12].

\section{Real-time quantitative PCR analysis for $B A A L C$}

From each patient and healthy subject $3 \mathrm{ml}$ of $\mathrm{PB}$ or $\mathrm{BM}$ samples were collected in sterile EDTA vacutainers. BAALC mRNA expression was normalized to the simultaneously analyzed glucose phosphate isomerase (GPI) gene. The relative BAALC expression was determined using the comparative cycle threshold (CT) method. Glucose phosphate isomerase (GPI) and BAALC were coamplified in the same tube using $1 \mu \mathrm{L}$ cDNA, $1 \times$ master mix (IQ Mix; BioRad, Munich, Germany), GPI probe (5'-HEX-TTCAGCTTGACCCTCAACACCAAC-TAMRA-3') with GPI forward (5'-TCTTCGATGCCAACAAGGAC-3') and reverse (5'-GCATCACGTCCTCCGTCAC-3') primers, and BAALC probe (5'-FAMCTCTTTTAGCCTCTGTGGTCTGAAGGCCATTAMRA-3') with BAALC forward (5'-GCCCTCTGACCCAGAAACAG-3') and reverse (5'-CTTTTGCAGGCATTCTCTTAGCA-3') primers. Reactions were performed using real-time PCR 7000 sequence detection system (Applied Biosystems, Foster City, USA). Positive and negative controls were included in all assays.

\section{Statistical analysis}

The statistical analysis of data was done by using excel program and SPSS (statistical package for social science) program (SPSS, Inc, Chicago, IL) version 16. Qualitative data were presented as frequency and percentage. Chi square test was used to compare groups. Quantitative data were presented as mean and standard deviation. For comparison between two groups; student t-test and Mann-whitney test (for non-parametric data) were used. Kaplan-Meier test was used for survival analysis and the statistical significance of differences among curves was determined by Log-Rank test. Prediction of survival was done using multivariate analysis. For gene expression quantification, we used the comparative $\mathrm{Ct}$ method. First, gene expression levels for each sample were normalized to the expression level of the housekeeping gene encoding GPI within a given sample $(\Delta \mathrm{Ct})$. Results were evaluated by using ${ }^{-{ }^{-\Delta C T}}$ method as relative gene expression values. N.B: $p$ is significant if $\leq 0.05$ at confidence interval $95 \%$.

\section{Results}

\section{$B A A L C$ expression and relationship with clinical, laboratory and molecular features}

ALL Patients were divided into $2 B A A L C$ expression groups; high $B A A L C$ expression (above median) and low BAALC expression (below median). The clinicohematological features differed in patients with high $B A A L C$ and those with low BAALC mRNA expression levels. Patients expressing high $B A A L C$ gene were significantly older $(P$ $=0.037)$, had higher WBC $(P=0.019)$, LDH concentration $(\mathrm{p}=0.007)$ 
Citation: Taalab MM, Fawzy IM, Goda EF, Salam EMA (2014) BAALC Gene Expression in Adult B-precursor Acute Lymphoblastic Leukemia: Impact on Prognosis. J Blood Disorders Transf 5: 220. doi: 10.4172/2155-9864.1000220

Page 3 of 6

and CD34 positivity $(p=0.011)$ compared with patients with low $B A A L C$ expression group. In addition, higher $B A A L C$ expression was associated with the presence of BCR-ABL $(P=0.011)$. There was no significant association between $B A A L C$ expression level and sex, hemoglobin concentration, platelet count, peripheral or marrow blasts, or immunophenotypic subgroups of B-precursor ALL. No other association was seen between $B A A L C$ expression and clinical features (Table 1).

\section{Outcome in B-precursor ALL patients with respect to $B A A L C$ expression}

Complete remission rate did not differ significantly between high and low BAALC expression groups $(p=0.120$ ), although patients with high $B A A L C$ expression showed a marginally significant lower CCR rate than low expression group ( $30 \%$ versus $43 \%$ respectively; $p=0.056$ ) and a higher incidence of primary resistant disease (34\% versus $14 \%$ respectively; $p=0.001)$. No influence on the relapse rate $(P=0.674)$ was observed with respect to $B A A L C$ expression. There were no significant differences regarding deaths in induction therapy $(P=0.203)$ between the two BAALC expression groups. Total mortality rates during the entire period of the study were higher in high $B A A L C$ expression group when compared to low expression group, but did not reach significant level $(P=0.063)$ (Table 2$)$.

When applying BAALC expression, age, marrow blasts, immunophenotypes and BCRABL as covariates for prediction of resistant disease, $B A A L C$ remained a predictive factor for primary therapy resistance $(P=0.003 ; \mathrm{OR}=3.133 ; 95 \%$ confidence interval [CI], 1.482-6.623), as well as immunophenotype (common versus pre B-ALL $(P=0.015$; OR= 2.326; 95\%CI, 1.431-7.956) (Table 3).

Overall survival (OS) was significantly shorter in patients with higher $B A A L C$ expression compared with those with lower $B A A L C$ expression (34.39 versus 50.11 months; 4 -year cumulative OS \%, 39.6\%, $53.6 \%$ respectively; $p=0.010$ ). In addition, high $B A A L C$ expression group showed significantly shorter DFS than those with low expression ( 27.93 versus 58.82 months; $51.7 \%$ versus $64.9 \%$ respectively; $\mathrm{p}<0.001$ ) (Figure 1). BAALC expression was independently predictive for OS and DFS in multivariate analysis $(\mathrm{p}=0.039, \mathrm{HR}=1.652,95 \% \mathrm{CI}=1.025-2.663$;

\begin{tabular}{|c|c|c|c|c|}
\hline & Total cases $(n=200)$ & Low expression group $(n=100)$ & High expression group $(n=100)$ & p \\
\hline Age (years) & $42.32 \pm 10.675$ & $40.75 \pm 10.240$ & $43.90 \pm 10.917$ & 0.037 \\
\hline $\begin{array}{l}\text { Sex Males } \\
\text { Females }\end{array}$ & $\begin{array}{l}106(53 \%) \\
94(47 \%)\end{array}$ & $\begin{array}{l}49(49 \%) \\
51(51 \%)\end{array}$ & $\begin{array}{l}57(57 \%) \\
43(43 \%)\end{array}$ & 0.257 \\
\hline Fever & $144(72.0 \%)$ & $76(76 \%)$ & $68(68 \%)$ & 0.208 \\
\hline Infection & $128(64.0 \%)$ & $66(66 \%)$ & $62(\%)$ & 0.556 \\
\hline Bleeding & $96(48.0 \%)$ & $45(45 \%)$ & $51(51 \%)$ & 0.396 \\
\hline Lymphadenopathy & $112(56.0 \%)$ & $53(53 \%)$ & $59(59 \%)$ & 0.393 \\
\hline Hepatomegaly & $84(42.0 \%)$ & $46(46 \%)$ & $38(38 \%)$ & 0.252 \\
\hline Splenomegaly & $100(50.0 \%)$ & $55(55 \%)$ & $45(45 \%)$ & 0.157 \\
\hline Mediastinal mass & $5(2.5 \%)$ & $2(2 \%)$ & $3(\%)$ & 0.651 \\
\hline WBC (X109/I) & $55.40 \pm 65.670$ & $44.50 \pm 44.052$ & $66.30 \pm 80.548$ & 0.019 \\
\hline $\mathrm{Hb}(\mathrm{g} / \mathrm{dl})$ & $9.5676 \pm 2.17064$ & $9.7754 \pm 2.07674$ & $9.3598 \pm 2.25185$ & 0.176 \\
\hline Platelets (X109/I) & $42.79 \pm 61.462$ & $37.17 \pm 51.071$ & $48.41 \pm 70.150$ & 0.197 \\
\hline Peripheral blasts (\%) & $53.98 \pm 21.502$ & $54.75 \pm 23.255$ & $53.21 \pm 19.680$ & 0.614 \\
\hline Marrow blasts (\%) & $69.44 \pm 20.734$ & $71.44 \pm 19.864$ & $67.44 \pm 21.483$ & 0.173 \\
\hline $\mathrm{LDH}(\mathrm{u} / \mathrm{l})$ & $1011.4 \pm 912.10$ & $837.43 \pm 635.88788$ & $1185.4 \pm 1098.53183$ & 0.007 \\
\hline $\begin{array}{l}\text { Immunophenotype } \\
\text { CD34 }\end{array}$ & $133(66.5 \%)$ & $58(58.0 \%)$ & $75(75.0 \%)$ & 0.011 \\
\hline $\begin{array}{l}\text { Immunophenotype subtypes } \\
\text { Pro B ALL } \\
\text { Common B ALL } \\
\text { Pre B ALL }\end{array}$ & $\begin{array}{c}16(8.0 \%) \\
144(72.0 \%) \\
40(20.0 \%)\end{array}$ & $\begin{array}{c}7(7.0 \%) \\
68(68.0 \%) \\
25(25.0 \%)\end{array}$ & $\begin{array}{c}9(9.0 \%) \\
76(76.0 \%) \\
15(15.0 \%)\end{array}$ & 0.202 \\
\hline $\begin{array}{l}\text { BCR-ABL } \\
\text { Positive } \\
\text { Negative }\end{array}$ & $\begin{array}{c}67(33.5 \%) \\
133(66.7 \%)\end{array}$ & $\begin{array}{l}25(25.0 \%) \\
75(75.0 \%)\end{array}$ & $\begin{array}{l}42(42.0 \%) \\
58(58.0 \%)\end{array}$ & 0.011 \\
\hline
\end{tabular}

Age, WBC, Hb, platelats, blasts, $\mathrm{LDH}$ and uric acid were presented by mean $\pm \mathrm{SD}$. Clinical presentations, immunophenotype and gender were presented by number and percentages. WBC: White Blood Cell Count; Hb: Hemoglobin Concentration; LDH: Lactate Dehydrogenase

Table 1: Clinical, laboratory and molecular characteristics at diagnosis according to BAALC expression in B-precursor ALL.

\begin{tabular}{|c|c|c|c|c|c|c|c|c|c|c|c|c|c|c|c|c|c|c|}
\hline \multirow{3}{*}{$\begin{array}{l}\text { BCR-ABL } \\
\text { Response }\end{array}$} & \multicolumn{6}{|c|}{ Total cases $(n=200)$} & \multicolumn{6}{|c|}{ BCR-ABL- $(n=133)$} & \multicolumn{6}{|c|}{ BCR-ABL+ $(n=67)$} \\
\hline & \multirow[t]{2}{*}{ No. } & \multicolumn{2}{|c|}{$\begin{array}{l}\text { Low BAALC } \\
\text { expression } \\
\text { group }(n=100)\end{array}$} & \multicolumn{2}{|c|}{$\begin{array}{c}\text { High } B A A L C \\
\text { expressio } \\
\text { group }(n=100)\end{array}$} & \multirow[t]{2}{*}{$\mathbf{p}$} & \multirow[t]{2}{*}{ No. } & \multicolumn{2}{|c|}{$\begin{array}{c}\text { Low BAALC } \\
\text { expression group } \\
(n=75)\end{array}$} & \multicolumn{2}{|c|}{$\begin{array}{l}\text { High } B A A L C \\
\text { expression } \\
\text { group }(n=58)\end{array}$} & \multirow[t]{2}{*}{$\mathbf{p}$} & \multirow[t]{2}{*}{ No } & \multicolumn{2}{|c|}{$\begin{array}{l}\text { Low BAALC } \\
\text { expression } \\
\text { group }(n=25)\end{array}$} & \multicolumn{2}{|c|}{$\begin{array}{l}\text { High } B A A L C \\
\text { expression } \\
\text { group }(n=42)\end{array}$} & \multirow[t]{2}{*}{$\mathbf{p}$} \\
\hline & & No & $\%$ & No & $\%$ & & & No & $\%$ & No & $\%$ & & & No & $\%$ & No & $\%$ & \\
\hline CR & 99 & 55 & 55 & 44 & 44 & 0.120 & 78 & 47 & 62.7 & 31 & 53.4 & 0.284 & 21 & 8 & 32.0 & 13 & 31.0 & 0.929 \\
\hline CCR & 73 & 43 & 43 & 30 & 30 & 0.056 & 62 & 38 & 50.7 & 24 & 41.4 & 0.287 & 11 & 5 & 20.0 & 6 & 14.3 & 0.541 \\
\hline Refractory & 48 & 14 & 14 & 34 & 34 & 0.001 & 32 & 13 & 17.3 & 19 & 32.8 & 0.039 & 16 & 1 & 4.0 & 15 & 35.7 & 0.003 \\
\hline Relapse & 26 & 12 & 12 & 14 & 14 & 0.674 & 16 & 9 & 12.0 & 7 & 12.1 & 0.990 & 10 & 3 & 12.0 & 7 & 16.7 & 0.604 \\
\hline Induction death & 53 & 31 & 31 & 22 & 22 & 0.203 & 23 & 15 & 20.0 & 8 & 13.8 & 0.348 & 31 & 16 & 64.0 & 15 & 35.7 & 0.025 \\
\hline Alive/ Died & $115 / 85$ & $64 / 36$ & $64 / 36$ & $51 / 49$ & $51 / 49$ & 0.063 & $96 / 37$ & $57 / 18$ & $76.0 / 24.0$ & $39 / 19$ & $67.2 / 32.8$ & 0.264 & $19 / 48$ & $7 / 18$ & $28.0 / 72.0$ & $12 / 30$ & $28.6 / 71.4$ & 0.960 \\
\hline
\end{tabular}

Table 2: Clinical outcome according to $B A A L C$ expression in $B C R-A B L^{-}$and $B C R-A B L^{+}$subgroups. 
(A)

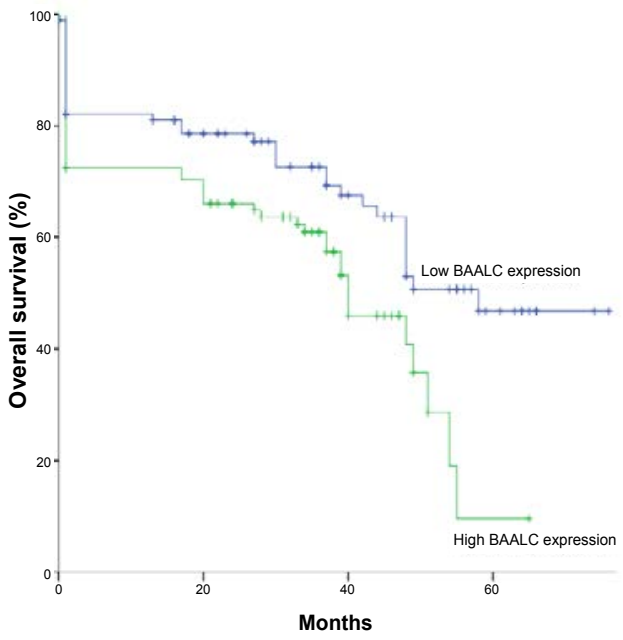

(B)

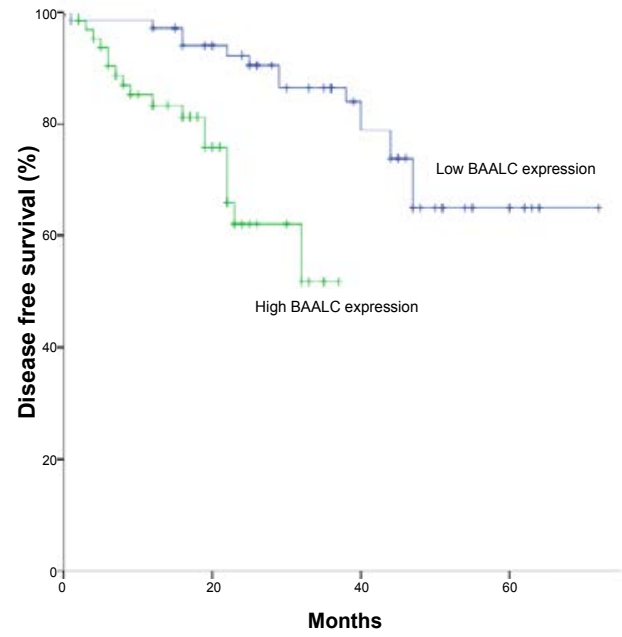

Figure 1: Survival times according to BAALC expression in adult B- precursor ALL patients. (A) OS and (B) DFS.

$p=0.002, \mathrm{HR}=1.214,95 \% \mathrm{CI}=1.013-4.368$ respectively). In addition, BCR-ABL is an independent unfavourable prognostic factor for OS $(p<0.001, \mathrm{HR}=2.749,95 \% \mathrm{CI}=1.124-4.940)$, as well as common B-ALL immunophenotype for DFS $(p<0.001, \mathrm{HR}=0.254,95 \% \mathrm{CI}=0.187-2.872)$ (Table 4).

\section{$B A A L C$ expression and outcome according to $\mathrm{BCR}-\mathrm{ABL}$ in B-precursor ALL patients}

All studied patients were stratified according to BCR-ABL into BCR-ABL- and BCR-ABL+ groups ( $\mathrm{n}=133,67$ respectively). We analyzed the prognostic impact of $B A A L C$ expression in these low- and high-risk subgroups of BCR-ABL B-precursor ALL. In the BCR-ABLgroup, no significant differences were obtained regarding $\mathrm{CR}, \mathrm{CCR}$, relapse, induction death and total mortality rates. Refractory disease was significantly higher in patients with high $B A A L C$ expression versus those with low BAALC expression ( $p=0.039)$. In addition, in the BCR$A B L+$ group, no significant differences were obtained regarding $C R$, CCR, relapse and total mortality rates. Resistance to chemotherapy was higher in patients expression high $B A A L C$ expression versus those with low $B A A L C$ expression group $(p=0.003)$. Death during induction therapy was significantly higher in low versus high $B A A L C$ expression groups $(p=0.025)$ (Table 2). Multivariate analysis showed that $B A A L C$ gene expression was independent risk factor for resistance to chemotherapy in BCR-ABL- and BCR-ABL+ groups $(\mathrm{OR}=2.359,95 \%$ $\mathrm{CI}=1.004-5.538, \mathrm{p}=0.049 ; \mathrm{OR}=2.672,95 \% \mathrm{CI}=1.824-3.326, \mathrm{p}=0.014$ respectively) (Table 5 ).

OS in $B C R-A B L^{-}$patients was shorter in high versus low $B A A L C$ expression group, although did not reach significant level (4-year OS: $58.5 \%, 66.2 \% ; 47.63,60.48$ months; $p=0.102$ ). Higher BAALC expression was significantly associated with an inferior DFS when compared to low $B A A L C$ expression group for $B C R-A B L^{-}$patients (4year DFS: $69.1 \%, 51.2 \%$; 60.36, 28.21 months $P<0.001)$. BCR-ABL+ patients showed no significant differences between $B A A L C$ expression groups for OS and DFS ( $p=0.991,0.671$ respectively) (Figure 2$)$. In the Cox regression analysis, $B A A L C$ expression was an independent adverse factor regarding DFS in BCR-ABL- group $(\mathrm{p}=0.001, \mathrm{HR}=3.774$, 95\% CI= 1.831-5.448) (Table 6).

\section{Discussion}

\begin{tabular}{|c|c|c|c|c|c|}
\hline \multirow{2}{*}{\multicolumn{2}{|c|}{ Refractory disease }} & \multirow{2}{*}{$p$} & \multirow{2}{*}{ OR } & \multicolumn{2}{|c|}{ 95.0\% C.I. } \\
\hline & & & & Lower & Upper \\
\hline \multicolumn{2}{|l|}{ Age (years) } & 0.644 & 1.008 & 0.976 & 1.041 \\
\hline \multicolumn{2}{|l|}{ Marrow blasts (\%) } & 0.349 & 0.992 & 0.975 & 1.009 \\
\hline \multirow{3}{*}{ Immunophenotype } & Pre & Referent & 1 & \multicolumn{2}{|c|}{-} \\
\hline & Common & 0.015 & 2.326 & 1.431 & 7.956 \\
\hline & Pro & 0.220 & 1.945 & 0.672 & 5.629 \\
\hline \multicolumn{2}{|c|}{ BCR-ABL (positive vs negative) } & 0.264 & 0.643 & 0.296 & 1.395 \\
\hline \multicolumn{2}{|c|}{ BAALC (high vs low) } & 0.003 & 3.133 & 1.482 & 6.623 \\
\hline
\end{tabular}

Table 3: Multivariate analysis for predicting chemotherapy resistance in all studied cases.

\begin{tabular}{|l|c|c|c|c|c|c|c|c|}
\hline \multirow{2}{*}{ Covariates } & \multicolumn{4}{|c|}{ OS } & \multicolumn{4}{c|}{ DFS } \\
\cline { 2 - 10 } & $\mathbf{P}$ & HR & $\begin{array}{c}\mathbf{9 5 \%} \text { Cl for } \\
\text { HR }\end{array}$ & P & HR & $\begin{array}{c}\mathbf{9 5 \%} \text { CI for } \\
\text { HR }\end{array}$ \\
\hline Age (years) & 0.081 & 0.982 & 0.961 & 1.002 & 0.819 & 0.996 & 0.964 & 1.030 \\
\hline Marrow blasts (\%) & 0.090 & 1.010 & 0.998 & 1.022 & 0.189 & 1.011 & 0.994 & 1.029 \\
\hline $\begin{array}{l}\text { Immunophenotype } \\
\text { Pro B ALL } \\
\text { Common B ALL }\end{array}$ & $\begin{array}{c}\text { Referent } \\
0.394\end{array}$ & 1 & - & - & Referent & 1 & - & - \\
\hline $\begin{array}{l}\text { Pre B ALL } \\
\text { BAALC expression } \\
\text { (high versus low) }\end{array}$ & 0.558 & 1.299 & 0.535 & 2.978 & $<0.001$ & 0.254 & 0.187 & 2.872 \\
\hline BCR-ABL & 0.039 & 1.652 & 1.025 & 2.663 & 0.002 & 1.214 & 1.013 & 4.368 \\
\hline
\end{tabular}

Table 4: Multivariate analysis for DFS and OS as dependent parameters studied with other covariates in all studied cases.

Acute lymphoblastic leukemia (ALL) remains one of the most challenging adult malignancies, especially with respect to therapy. Immunophenotyping, cytogenetic-molecular studies [12]. However, most of the studies focused on children and therefore a deep molecular characterization of adults is still challenging, especially for those cases lacking high-risk markers (eg, $B C R-A B L$ ). In this study, we have evaluated the prognostic significance of $B A A L C$ expression in B-precursor ALL.

In B-precursor ALL, higher BAALC expression was associated with older age, higher WBC, $\mathrm{LDH}$ concentration and $B C R-A B L$, which are known risk factors in accordance with others [13]. However, no significant differences were found between low and high $B A A L C$ 
Citation: Taalab MM, Fawzy IM, Goda EF, Salam EMA (2014) BAALC Gene Expression in Adult B-precursor Acute Lymphoblastic Leukemia: Impact on Prognosis. J Blood Disorders Transf 5: 220. doi: 10.4172/2155-9864.1000220

Page 5 of 6

\begin{tabular}{|c|c|c|c|c|c|c|c|c|c|}
\hline \multirow{3}{*}{\multicolumn{2}{|c|}{$\begin{array}{l}\text { BCR-ABL } \\
\text { Covariates } \\
\text { Age }\end{array}$}} & \multicolumn{4}{|c|}{ BCR-ABL- $(n=140)$} & \multicolumn{4}{|c|}{$B C R-A B L^{+}(n=60)$} \\
\hline & & \multirow{2}{*}{$\begin{array}{c}p \\
0.930\end{array}$} & \multirow{2}{*}{$\begin{array}{c}\text { OR } \\
0.998\end{array}$} & \multicolumn{2}{|c|}{$95.0 \%$ C.I } & \multirow{2}{*}{$\begin{array}{c}p \\
0.922\end{array}$} & \multirow{2}{*}{$\begin{array}{c}\text { OR } \\
1.003\end{array}$} & \multicolumn{2}{|c|}{$95.0 \%$ C.I } \\
\hline & & & & 0.957 & 1.041 & & & 0.943 & 1.068 \\
\hline Marrow blasts & & 0.080 & 0.982 & 0.963 & 1.002 & 0.121 & 1.035 & 0.991 & 1.082 \\
\hline \multirow{3}{*}{ Immunophenotype } & Pre & Referent & 1 & - & & Referent & 1 & - & \\
\hline & Common & 0.196 & 0.332 & 0.062 & 1.766 & 0.018 & 0.075 & 0.009 & 0.638 \\
\hline & Pro & 0.119 & 0.232 & 0.037 & 1.454 & 0.999 & 0.003 & 0.001 & 0.276 \\
\hline \multicolumn{2}{|l|}{$B A A L C$ (high vs low) } & 0.049 & 2.359 & 1.004 & 5.538 & 0.014 & 2.672 & 1.824 & 3.326 \\
\hline
\end{tabular}

Table 5: Multivariate analysis for predicting chemotherapy resistance in in $B C R-A B L$-and $B C R$ - $A B L+$ subgroups.

(A)

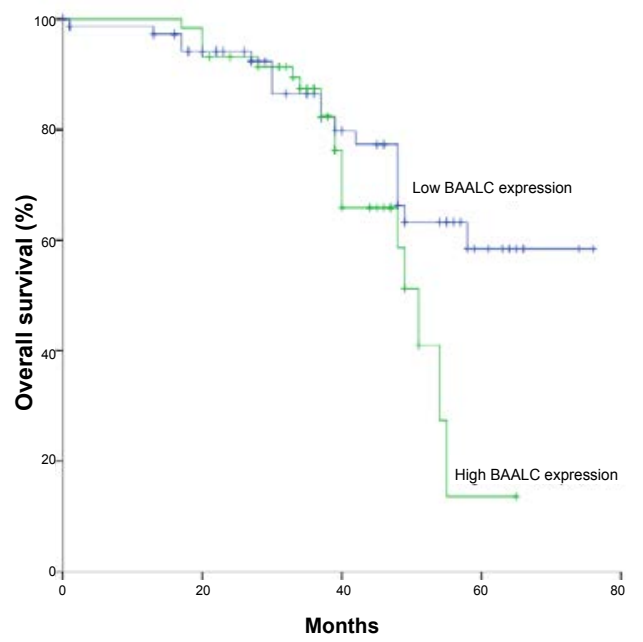

OS in BCR-ABL negative

(C)

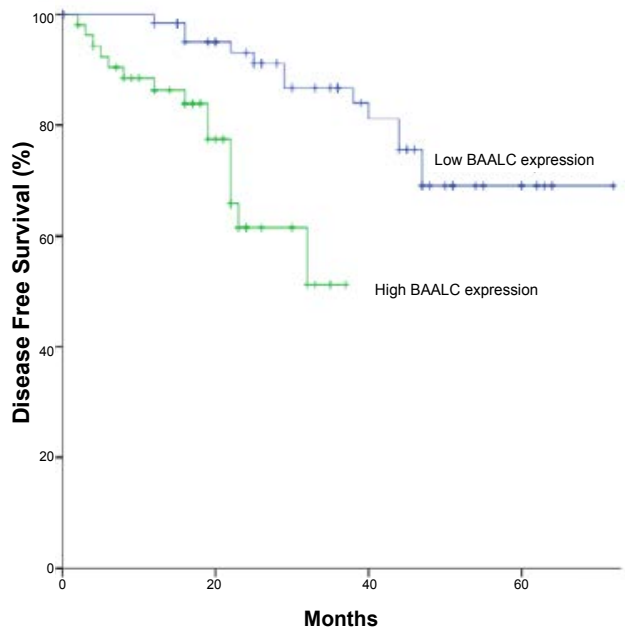

DFS in BCR-ABL negative
(B)

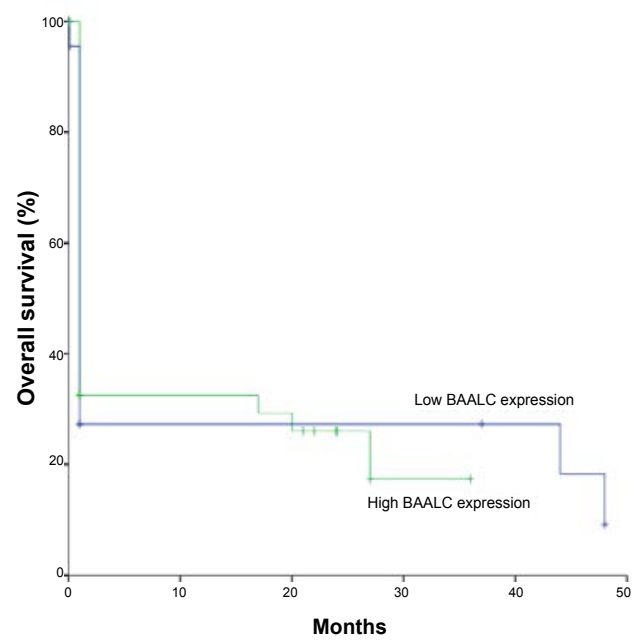

OS in BCR-ABL positive

(D)

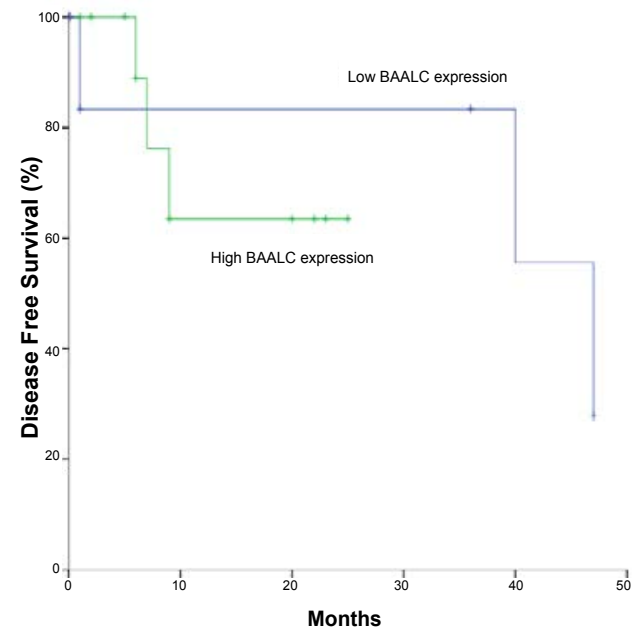

DFS in BCR-ABL positive

Figure 2: Survival times according to $B C R-A B L$ status and $B A A L C$ expression in adult $B-$ precursor $A L L$ patients. $(A, B) O S$ in $B C R-A B L^{-}$negative, $B C R-A B L^{+}$ respectively, $(C, D)$ DFS in $B C R-A B L-$ negative and $B C R-A B L+$ respectively.

expression groups regarding clinical presentations, sex, hemoglobin concentration, platelet count, peripheral or marrow blasts, or immunophenotypic subgroups of B-precursor ALL.

Patients with low BAALC gene expression continued $\mathrm{CR}$ at a rate marginally significantly higher than those with high $B A A L C$ gene expression. High $B A A L C$ gene expression group confer higher resistance to chemotherapy than those with low $B A A L C$ gene expression in agreement with Kohnl [13]. Total mortality rate was marginally significantly higher in high versus low $B A A L C$ gene expression groups. 
Citation: Taalab MM, Fawzy IM, Goda EF, Salam EMA (2014) BAALC Gene Expression in Adult B-precursor Acute Lymphoblastic Leukemia: Impact on Prognosis. J Blood Disorders Transf 5: 220. doi: 10.4172/2155-9864.1000220

Page 6 of 6

\begin{tabular}{|c|c|c|c|c|c|c|c|c|c|c|}
\hline & \multirow{2}{*}{\multicolumn{2}{|c|}{$\begin{array}{c}\text { BCR-ABL } \\
\text { Covariates }\end{array}$}} & \multicolumn{4}{|c|}{ BCR-ABL- $(n=140)$} & \multicolumn{4}{|c|}{ BCR-ABL ${ }^{+}(n=60)$} \\
\hline & & & \multirow{2}{*}{$\begin{array}{c}p \\
0.726\end{array}$} & \multirow{2}{*}{$\begin{array}{c}\text { HR } \\
1.006\end{array}$} & \multicolumn{2}{|c|}{$95.0 \%$ C.I } & \multirow{2}{*}{$\begin{array}{c}p \\
0.378\end{array}$} & \multirow{2}{*}{$\begin{array}{c}\text { HR } \\
0.986\end{array}$} & \multicolumn{2}{|c|}{$95.0 \%$ C.I } \\
\hline \multirow{7}{*}{ os } & Age & & & & 0.974 & 1.038 & & & 0.955 & 1.017 \\
\hline & Marrow blasts & & 0.064 & 1.016 & 0.999 & 1.033 & 0.367 & 1.009 & 0.990 & 1.029 \\
\hline & Immuno-phenotype & Pre & Referent & 1 & - & Referent & 1 & - & & \\
\hline & & Common & 0.913 & 2.511 & 0.045 & 4.196 & 0.729 & 0.830 & 0.289 & 2.382 \\
\hline & & Pro & 0.922 & 1.183 & 0.047 & 3.037 & 0.465 & 0.580 & 0.135 & 2.501 \\
\hline & \multicolumn{2}{|l|}{$B A A L C$ (high vs low) } & 0.152 & 1.654 & 0.831 & 3.290 & 0.743 & 1.123 & 0.563 & 3.240 \\
\hline & Age & & 0.954 & 0.999 & 0.965 & 1.034 & 0.246 & 0.820 & 0.586 & 1.147 \\
\hline \multirow{5}{*}{ DFS } & Marrow blasts & & 0.391 & 1.008 & 0.990 & 1.026 & 0.109 & 1.086 & 0.982 & 1.200 \\
\hline & \multirow{3}{*}{ Immuno-phenotype } & Pre & Referent & 1 & - & Referent & 1 & - & & \\
\hline & & Common & 0.920 & 2.110 & 0.876 & 5.919 & 0.215 & 2.440 & 0.212 & 4.579 \\
\hline & & Pro & 0.926 & 1.362 & 0.285 & 4.349 & 0.735 & 1.027 & 0.436 & 5.981 \\
\hline & \multicolumn{2}{|l|}{$B A A L C$ (high vs low) } & 0.001 & 3.774 & 1.831 & 5.448 & 0.666 & 1.794 & 0.126 & 5.528 \\
\hline
\end{tabular}

Table 6: Multivariate analysis for DFS and OS as dependent parameters studied with other covariates according to BCR-ABL status

This may be due to lower CCR rate, higher resistance to chemotherapy, more aggressive disease having more immature cells. In multivariate analysis, high $B A A L C$ expression retains its high risk for primary therapy failure, in accordance with other reports [13].

When stratifying all patients according to BCR-ABL, 133 cases were BCR-ABL negative and 67 patients were BCR-ABL+. High $B A A L C$ gene expression group had significantly higher resistance to chemotherapy in $\mathrm{BCR}-\mathrm{ABL}$ and $\mathrm{BCR}-\mathrm{ABL}^{+}$groups. In multivariate analysis, high $B A A L C$ gene expression is still independent prognostic factor for resistance to chemotherapy in BCR-ABL and BCR-ABL ${ }^{+}$ groups.

In addition, higher $B A A L C$ expression was independently predictive for DFS and OS all patients and for DFS in $B C R-A B L^{-}$patients.

In previous studies, high BAALC expression was associated with an unfavorable outcome and inferior long-term survival in adult CN-AML and T-ALL $[9,14,15]$. Thus, BAALC characterizes a more aggressive, immature, highly proliferative, and chemoresistant leukemic phenotype.

Our data suggest that BAALC may identify patients with an immature, chemoresistant leukemic phenotype associated with an unfavorable outcome and shorter survival of adult B-precursor ALL.

$B A A L C$ expression could better discriminate patients into various prognostic groups and identify patients who might benefit from doseintensified induction chemotherapy.

Stratification of adult B- precursor ALL into 2 distinctive groups of patients with outcome and survival characteristics, might in the future facilitate treatment stratification for adult B-precursor ALL.

\section{References}

1. Pui CH, Robison LL, Look AT (2008) Acute lymphoblastic leukemia. Lancet 354: 166-178.

2. Marks DI (2010) Treating the "older" adult with acute lymphoblastic leukemia Hematology Am Soc Hematol Educ Program 2010: 13-20.

3. Cobaleda C, Sánchez-García I (2009) B-cell acute lymphoblastic leukaemia: towards understanding its cellular origin. Bioessays 31: 600-609.

4. Gökbuget N, Hoelzer D (2009) Treatment of adult acute lymphoblastic leukemia. Semin Hematol 46: 64-75

5. Gomes AM, Soares MV, Ribeiro P, Caldas J, Póvoa V, et al. (2014) Adult B-cell acute lymphoblastic leukemia cells display decreased PTEN activity and constitutive hyperactivation of PI3K/Akt pathway despite high PTEN protein levels. Haematologica.
6. Zhou Y, Slack R, Jorgensen JL, Wang SA, Rondon G, et al. (2014) The Effect of Peritransplant Minimal Residual Disease in Adults With Acute Lymphoblastic LeukemiaUndergoing Allogeneic Hematopoietic Stem Cell Transplantation. Clin Lymphoma Myeloma Leuk.

7. Faderl S, Jeha S, Kantarjian HM (2003) The biology and therapy of adult acute lymphoblastic leukemia. Cancer 98: 1337-1354.

8. Tanner SM, Austin JL, Leone G, Rush LJ, Plass C, et al. (2001) BAALC the human member of a novel mammalian neuroectoderm gene lineage, is implicated in hematopoiesis and acute leukemia. Proc Natl Acad Sci U S A 98: 13901-13906.

9. Baldus CD, Tanner SM, Ruppert AS, Whitman SP, Archer KJ, et al. (2003) $B A A L C$ expression predicts clinical outcome of de novo acute myeloid leukemia patients with normal cytogenetics: a Cancer and Leukemia Group B Study. Blood 102: 1613-1618.

10. Baldus CD, Martus P, Burmeister T, Schwartz S, Gökbuget N, et al. (2007) Low ERG and BAALC expression identifies a new subgroup of adult acute T-lymphoblastic leukemia with a highly favorable outcome. J Clin Oncol 25: 3739-3745.

11. Heesch S, Schlee C, Neumann M, Stroux A, Kühnl A, et al (2010). BAALCassociated gene expression profiles define IGFBP7 as a novel molecular marker in acute leukemia. Leukemia. 24: 1429-1436.

12. Faderl S, O'Brien S, Pui CH, Stock W, Wetzler M, et al. (2010) Adult acute lymphoblastic leukemia: concepts and strategies. Cancer 116: 1165-1176.

13. Kühnl A, Gökbuget N, Stroux A, Burmeister T, Neumann M, et al. (2010) High BAALC expression predicts chemoresistance in adult B-precursor acute lymphoblastic leukemia. Blood 115: 3737-3744.

14. Langer C, Radmacher MD, Ruppert AS, Whitman SP, Paschka P, et al. (2008) High BAALC expression associates with other molecular prognostic markers, poor outcome, and a distinct gene-expression signature in cytogenetically normal patients younger than 60 years with acute myeloid leukemia: a Cancer and Leukemia Group B (CALGB) study. Blood 111: 5371-5379.

15. Baldus CD, Thiede C, Soucek S, Bloomfield CD, Thiel E, et al. (2006) BAALC expression and FLT3 internal tandem duplication mutations in acute myeloid leukemia patients with normal cytogenetics: prognostic implications. J Clin Oncol 24: 790-797.

Citation: Taalab MM, Fawzy IM, Goda EF, Salam EMA (2014) BAALC Gene Expression in Adult B-precursor Acute Lymphoblastic Leukemia: Impact on Prognosis. J Blood Disorders Transf 5: 220. doi: 10.4172/2155-9864.1000220 